\title{
Creating Multi-Sided Rational Bézier Surfaces using Base Points
}

\author{
Joe Warren \\ Department of Computer Science \\ Rice University *
}

May 1990

\begin{abstract}
Rational Bézier surfaces provide an effective tool for geometric design. One aspect of the theory of rational surfaces that is not well-understood is what happens when a rational parameterization takes on the value $\left(\frac{0}{0}, \frac{0}{0}, \frac{0}{0}\right)$ for some parameter value. Such parameter values are called base points of the parameterization. Base points can be introduced into a rational parameterization in Bézier form by setting weights of appropriate control points to zero. By judiciously introducing base points, one can create parameterizations of four-, five- and six-sided surface patches using rational Bézier surfaces defined over triangular domains. Subdivision techniques allow rendering and smooth meshing of such surfaces. Properties of base points also lead to a new understanding of incompatible edge twist methods such as Gregory's patch.
\end{abstract}

\section{Introduction}

Triangular parametric surface patches are an important tool in geometric modeling. Mathematically, a surface patch $S$ is the image of a domain triangle $T$ under some parametric mapping. If $T$ has vertices $\mathbf{p}_{1}, \mathbf{p}_{2}$, and $\mathbf{p}_{3}$, any point $\mathbf{q}$ in $T$ can be expressed uniquely in the form $\mathbf{q}=\alpha \mathbf{p}_{1}+\beta \mathbf{p}_{2}+\gamma \mathbf{p}_{3}$ where $\alpha, \beta, \gamma \geq 0$ and $\alpha+\beta+\gamma=1$. The components of the triple $(\alpha, \beta, \gamma)$ are called the barycentric coordinates of $\mathbf{q}$ with respect to $\mathbf{p}_{1}, \mathbf{p}_{2}$, and $\mathbf{p}_{3}$.

One popular class of parametric surface patches uses polynomial maps. A triangular Bézier surface of parametric degree $n$ has the form:

$$
\mathbf{p}=\sum_{\substack{i, j, k>0 \\ i+j+k=n}} \mathbf{p}_{i j k} B_{i j k}(\alpha, \beta, \gamma),
$$

*Supported in part by NSF grants IRI 88-10747 and CCR 89-03431 
where $B_{i j k}$ are the bivariate Bernstein basis functions of degree $n$. These basis functions have the form:

$$
B_{i j k}(\alpha, \beta, \gamma)=\frac{n !}{i ! j ! k !} \alpha^{i} \beta^{j} \gamma^{k}
$$

The points $\mathbf{p}_{i j k}=\left(x_{i j k}, y_{i j k}, z_{i j k}\right)$ are called control points. These control points are linked together to form a control polyhedron. The shape of the surface patch $S$ and geometry of its corresponding control polyhedron are closely related, which has contributed to the popularity of Bézier patches in geometric design. Farin has summarized many of their properties [Far88].

Parameterizations such as equation 1 generate Cartesian coordinates of points in three dimensional affine space. One drawback of this representation is that simple surfaces like spheres and cylinders cannot be represented in this form. Fortunately, such surfaces can be included by extending the parameterization to generate points in three dimensional projective space. Points in projective space are represented using homogeneous coordinates. Homogeneous coordinates are tuples of the form $(x, y, z, w)$, and have the property that $(k x, k y, k z, k w)$ denotes the same point for all $k \neq 0$. If $w \neq 0$, the point $(x, y, z, w)$ in homogeneous coordinates represents the point $(x / w, y / w, z / w)$ in affine space.

We can define a larger class of surfaces with homogeneous coordinates. A rational Bézier surface of parametric degree $n$ has the form:

$$
\left(\begin{array}{c}
\mathbf{p} \\
w
\end{array}\right)=\sum_{\substack{i, j, k>0 \\
i+j+k=n}}\left(\begin{array}{c}
w_{i j k} \mathbf{p}_{i j k} \\
w_{i j k}
\end{array}\right) B_{i j k}(\alpha, \beta, \gamma)
$$

Points on the surface patch are represented in homogeneous coordinates $(\mathbf{p}, w)=(x, y, z, w)$. The points $\mathbf{p}_{i j k}=\left(x_{i j k}, y_{i j k}, z_{i j k}\right)$ are control points, and associated with each is a weight $w_{i j k}$. The set of all control points and their weights forms a weighted control polyhedron.

In the rational Bézier form, the weights $w_{i j k}$ are usually required to be nonnegative so that the resulting surface patch lies in the convex hull of its defining control points. With this requirement, the value of $w$ is always nonnegative. A point $(x, y, z, w)$ with $w>0$ is at finite distance and has Cartesian coordinates $(x / w, y / w, z / w)$. However, if $w$ is zero, then either the the point is located at infinity, when one of $x, y, z$ is nonzero, or the $x, y$, and $z$ values simultaneously vanish. This paper investigates what happens in the latter case; the effect on the resulting surface is surprising. 


\section{Base Points}

Parameter values for which $(x, y, z, w)=(0,0,0,0)$ are called base points of the parameterization. Little is known about the interaction of base points and geometric design. Gregory has used base points in his construction of a surface patch that smoothly interpolates $C^{1}$ boundary data [Gre74]. Gregory's patch is defined using a special collection of rational basis functions that evaluate to $\frac{0}{0}$ at vertices of the parametric domain and thus introduce base points in the resulting parameterization. Understanding the effect of the base points introduced by these rational functions leads to a new description of Gregory's construction, in terms of the properties of weights and control points. In his thesis, Chionh explores the relationship between base points of a rational surface and its equivalent implicitly defined surface [Chi90]. However, he uses the monomial basis representation of polynomials in his investigation. By expressing the parameterization in the Bernstein basis, we gain much greater insight into the effect of base points.

Introducing a base point into the parameterization of a rational Bézier surface is simple. For example, setting the weight $w_{00 n}$ to zero causes $x, y, z$, and $w$ to evaluate to zero at the parameter value $(0,0,1)$. What is the behavior of this rational Bézier surface near the parameter value $(0,0,1)$ ? As we shall see, the behavior of this surface is easily described in terms of neighboring control points.

\subsection{Base Points and Rational Curves}

We first consider the effect of base points on the behavior of rational curves. That analysis leads to a better understanding of the behavior of rational surfaces with base points. A rational Bézier curve of parametric degree $n$ has the form

$$
\left(\begin{array}{c}
\mathbf{p} \\
w
\end{array}\right)=\sum_{\substack{i, j \geq 0 \\
i+j=n}}\left(\begin{array}{c}
w_{i j} \mathbf{p}_{i j} \\
w_{i j}
\end{array}\right) B_{i j}(\alpha, \beta)
$$

where

$$
B_{i j}(\alpha, \beta)=\frac{n !}{i ! j !} \alpha^{i} \beta^{j}
$$

Dividing by $w$ yields affine points on the curve of the form:

$$
\frac{\mathbf{p}}{w}=\frac{\sum_{i, j \geq 0 ; i+j=n} w_{i j} \mathbf{p}_{i j} B_{i j}(\alpha, \beta)}{\sum_{i, j \geq 0 ; i+j=n} w_{i j} B_{i j}(\alpha, \beta)} .
$$


If $w_{0 n}=0$, then a factor of $\alpha$ can be canceled from both the numerator and denominator of equation 3. The result is a rational Bézier curve of parametric degree $n-1$ with control points $\hat{\mathbf{p}}_{i j}$ and weights $\hat{w}_{i j}$. These control points and weights satisfy the relation:

$$
\left(\begin{array}{c}
\hat{\mathbf{p}}_{i j} \\
\hat{w}_{i j}
\end{array}\right)=\left(\begin{array}{c}
\mathbf{p}_{(i+1) j} \\
\frac{n}{i+1} w_{(i+1) j}
\end{array}\right)
$$

for $i, j \geq 0 ; i+j=n-1$.

More generally, setting $w_{n 0}, w_{(n-1) 1}, \ldots, w_{(n-i) i}$ to zero produces a rational Bézier curve of degree $n-(i+1)$ whose control points are those that originally had nonzero weight. This construction can be applied to both ends of a curve. Figure 1 shows a rational quintic Bézier curve whose first and last control points have a zero weight. The Bézier curve shown is actually a cubic defined by the middle four control points.

\subsection{Base Points and Rational Surfaces}

In the curve case, introducing a base point by setting $w_{0 n}=0$ results in rational curve defined by the remaining control points. In the surface case, we examine the effect on the resulting rational surface of setting the weight $w_{00 n}=0$ to zero. To understand this effect, we first observe that any triangular patch can be reparameterized as a degenerate rectangular surface patch under the following transformation.

$$
\begin{gathered}
\lambda=\frac{\beta}{\alpha+\beta}, \\
\delta=1-\gamma .
\end{gathered}
$$

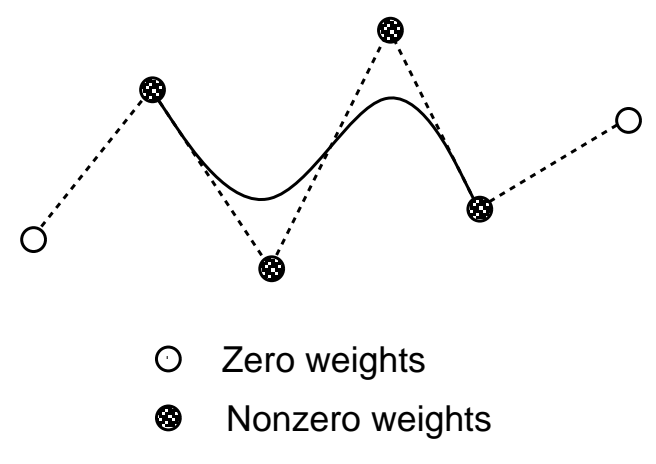

Figure 1: A rational quintic whose first and last control points have zero weight 


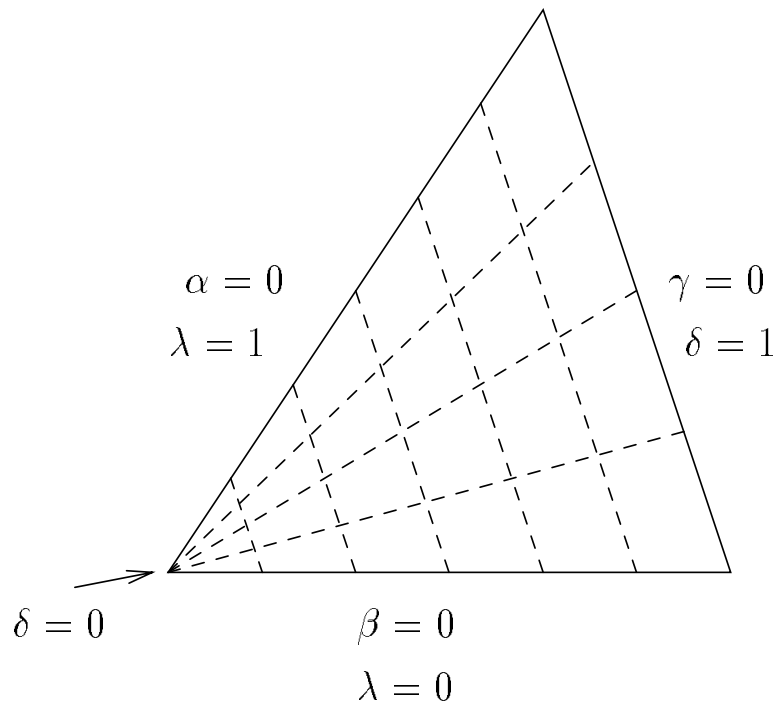

Figure 2: Transforming barycentric coordinates to Cartesian coordinates

This transformation maps barycentric coordinates $(\alpha, \beta, \gamma)$ to Cartesian coordinates $(\lambda, \delta)$. Figure 2 shows this transformation. The dashed lines are new Cartesian coordinates on the original domain triangle. Mathematically, this transformation stretches the vertex with barycentric coordinates $(0,0,1)$ to the edge $\delta=0$ in Cartesian coordinates. The inverse transformation is

$$
\begin{aligned}
\alpha & =(1-\lambda) \delta, \\
\beta & =\lambda \delta, \\
\gamma & =1-\delta .
\end{aligned}
$$

Applying the transformation of equation 6 to the definition of a rational triangular surface in equation 2 yields a tensor product surface defined over the unit square $0 \leq \lambda, \delta \leq 1$.

For example, consider a triangular rational quadratic Bézier surface:

$$
\begin{aligned}
\left(\begin{array}{c}
\mathbf{p} \\
w
\end{array}\right)= & \left(\begin{array}{c}
w_{002} \mathbf{p}_{002} \\
w_{002}
\end{array}\right) \gamma^{2}+\left(\begin{array}{c}
w_{101} \mathbf{p}_{101} \\
w_{101}
\end{array}\right) 2 \alpha \gamma+\left(\begin{array}{c}
w_{011} \mathbf{p}_{011} \\
w_{011}
\end{array}\right) 2 \beta \gamma+ \\
& \left(\begin{array}{c}
w_{200} \mathbf{p}_{200} \\
w_{200}
\end{array}\right) \alpha^{2}+\left(\begin{array}{c}
w_{110} \mathbf{p}_{110} \\
w_{110}
\end{array}\right) 2 \alpha \beta+\left(\begin{array}{c}
w_{020} \mathbf{p}_{020} \\
w_{020}
\end{array}\right) \beta^{2} .
\end{aligned}
$$

Applying transformation 6 to this triangular surface yields the following tensor product 
rational quadratic surface:

$$
\begin{aligned}
\left(\begin{array}{l}
\mathbf{p} \\
w
\end{array}\right)= & \left(\begin{array}{c}
w_{002} \mathbf{p}_{002} \\
w_{002}
\end{array}\right)(1-\delta)^{2}+\left(\left(\begin{array}{c}
w_{101} \mathbf{p}_{101} \\
w_{101}
\end{array}\right)(1-\lambda)+\left(\begin{array}{c}
w_{011} \mathbf{p}_{011} \\
w_{011}
\end{array}\right) \lambda\right)(2 \delta(1-\delta))+ \\
& \left(\left(\begin{array}{c}
w_{200} \mathbf{p}_{200} \\
w_{200}
\end{array}\right)(1-\lambda)^{2}+\left(\begin{array}{c}
w_{110} \mathbf{p}_{110} \\
w_{110}
\end{array}\right) 2(1-\lambda) \lambda+\left(\begin{array}{c}
w_{020} \mathbf{p}_{020} \\
w_{020}
\end{array}\right) \lambda^{2}\right) \delta^{2} .
\end{aligned}
$$

The expressions $w_{002} \mathbf{p}_{002}$ and $w_{101} \mathbf{p}_{101}(1-\lambda)+w_{011} \mathbf{p}_{011} \lambda$ can be degree-raised to quadratics to bring the patch into standard tensor product form.

Tensor product Bézier surfaces can be viewed as rational combinations in $\lambda$ of rational Bézier curves in $\delta$. Before setting the weight $w_{002}$ to zero, all control points on the $\delta=0$ boundary have value $\mathbf{p}_{002}$. The boundary curve is collapsed to a single point. The effect of setting $w_{002}$ to zero is the deletion of all the control points on the $\delta=0$ boundary, producing another tensor product surface. After deleting these control points, the boundary curve is no longer a point but a line connecting $\mathbf{p}_{10(n-1)}$ and $\mathbf{p}_{01(n-1)}$. The triangular surface has become four-sided. The remaining control points for the surface remain unchanged, but with their weights modified in a way consistent with equation 4, where the tensor product surface is treated as a collection of rational curves in $\delta$.

Setting $w_{002}=0$ in the previous example deletes all basis functions containing the factor $(1-\delta)^{2}$ and introduces a common factor of $\delta$ among all the parametric equations. Dividing by $\delta$ yields a surface patch of the form:

$$
\begin{aligned}
\left(\begin{array}{l}
\mathbf{p} \\
w
\end{array}\right)= & \left(\left(\begin{array}{c}
2 w_{101} \mathbf{p}_{101} \\
2 w_{101}
\end{array}\right)(1-\lambda)+\left(\begin{array}{c}
2 w_{011} \mathbf{p}_{011} \\
2 w_{011}
\end{array}\right) \lambda\right)(1-\delta)+ \\
& \left(\left(\begin{array}{c}
w_{200} \mathbf{p}_{200} \\
w_{200}
\end{array}\right)(1-\lambda)^{2}+\left(\begin{array}{c}
w_{110} \mathbf{p}_{110} \\
w_{110}
\end{array}\right) 2(1-\lambda) \lambda+\left(\begin{array}{c}
w_{020} \mathbf{p}_{020} \\
w_{020}
\end{array}\right) \lambda^{2}\right) \delta .
\end{aligned}
$$

More generally, if all weights $w_{i j k}$ for which $i+j<m$ are set to zero $(m<n-1)$, the result of applying transformation 6 to a triangular rational Bézier surface is a four-sided patch. Three of the sides of the patch are images of the edges of the original domain triangle. The fourth side, introduced by transformation 6 , is a rational Bézier curve of degree $m$ with control points $\mathbf{p}_{i j k}$ where $i+j=m$. 


\section{$3 \quad$ Geometric Design using Base Points}

\subsection{Creating Multi-Sided Patches}

Introducing base points by setting weights to zero produces four-sided patches that are images of domain triangles. The technique can be generalized to create five- and six-sided patches by treating each vertex of the triangular domain independently. Various methods for creating multiple-sided patches have been presented [Gre83a, Gre83b, HK84, LD89, Sab83], but our approach is distinct from these previous approaches in that the patches are created using the properties of triangular Bézier surfaces. Extra sides are added to the patches by setting the weights of an appropriate collection of control points to zero.

Let $m_{i}, m_{j}, m_{k} \geq 0$ satisfy $m_{i}+m_{j}, m_{i}+m_{k}, m_{j}+m_{k}<n$. Given a triangular rational Bézier surface of degree $n$, setting weights $w_{i j k}=0$ for all $i+j<m_{k}$ creates a new boundary curve of degree $m_{k}$ corresponding to the vertex $(0,0,1)$ in the original domain triangle. Setting $w_{i j k}=0$ for all $i+k<m_{j}$ and $w_{i j k}=0$ for all $j+k<m_{i}$ creates new boundary curves of degrees $m_{j}$ and $m_{i}$ respectively. These curves are the images of vertices $(0,1,0)$ and $(1,0,0)$ in the original domain triangle. If each of $m_{i}, m_{j}, m_{k}$ is greater than zero, the result is a six-sided patch. If only two of the three are greater than zero the result is a five-sided patch. Figure 3 illustrates the six-sided patch that results when $n=4$ and $m_{i}=m_{j}=m_{k}=1$. Note that the boundary curves that are the images of the original domain triangle now have degrees $n-m_{i}-m_{j}, n-m_{i}-m_{k}$, and $n-m_{j}-m_{k}$.

\subsection{Removal of Base Points from the Parameterization}

Mathematically, a five or six-sided surface patch arising from the previous construction is well-defined. However, from a computational viewpoint, there is still the question of how to deal with the rational parameterization near the base points. The conversion from barycentric coordinates to Cartesian coordinates provides the answer in the four-sided case. However, in the five- or six-sided case, this approach does not work. A solution is to subdivide the domain triangle into four subtriangles. The image of the middle subtriangle is triangular patch. The image of a subtriangle touching a vertex is either a three-sided or a four-sided patch depending on whether the weight for the control point at that vertex has been set to zero. Figure 4 illustrates this solution. 

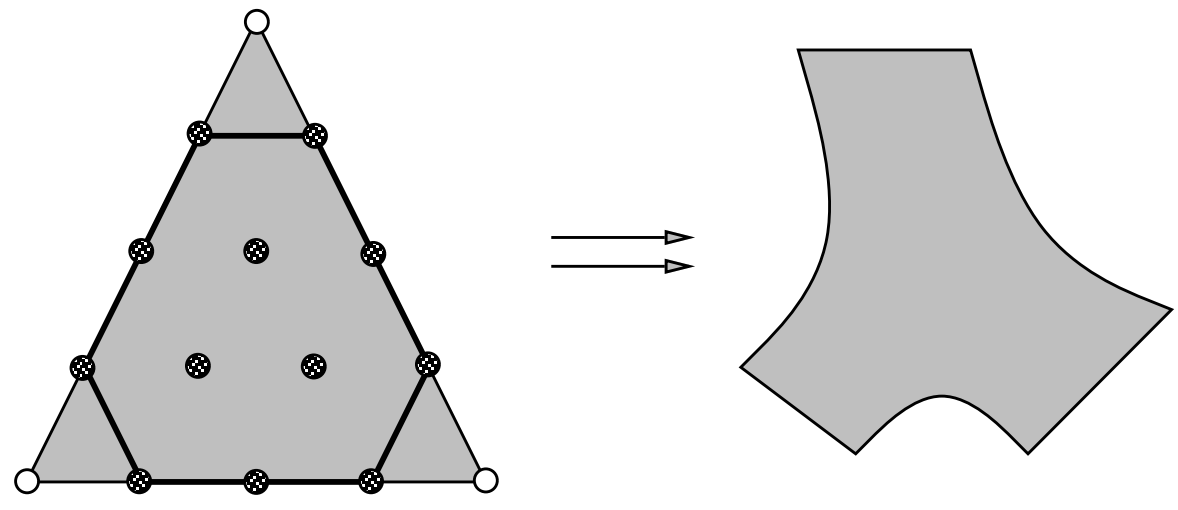

Zero weights

* Nonzero weights

- Newton polygon

FiguRE 3: Transforming a domain triangle into an $n$-sided patch
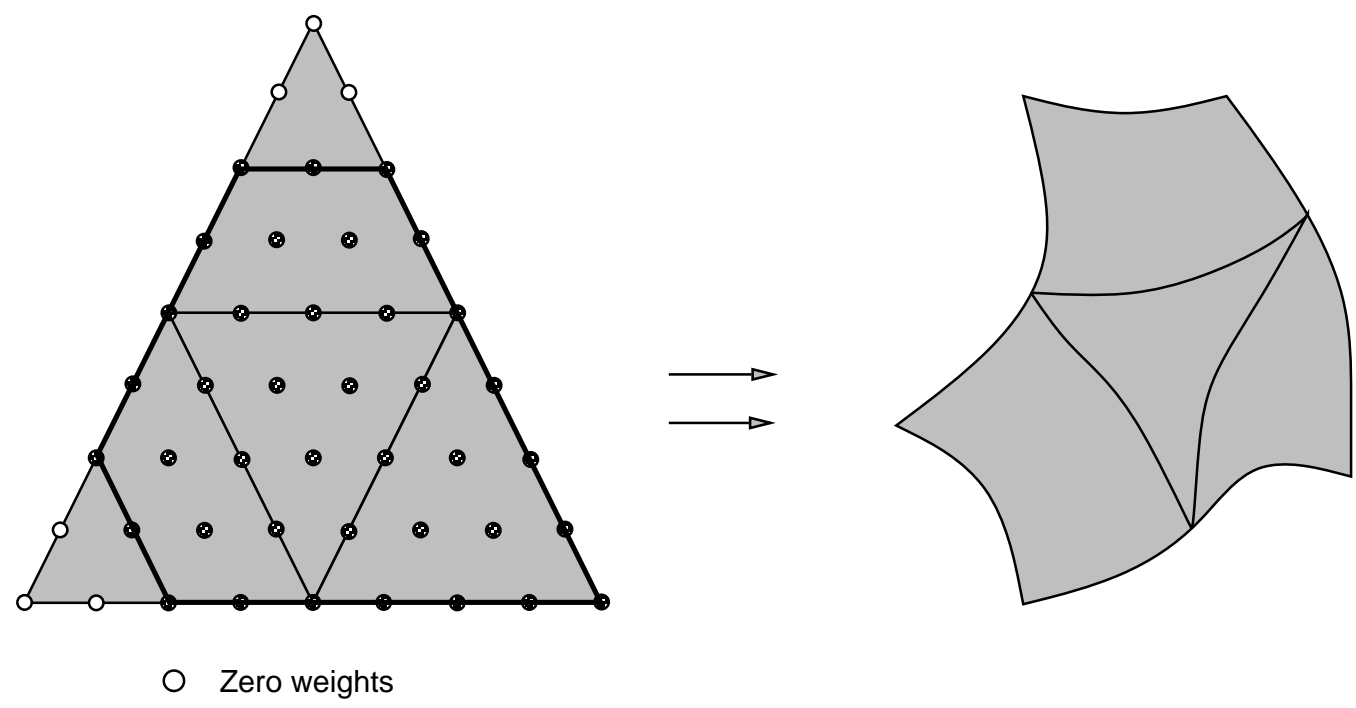

(2) Nonzero weights

FiguRE 4: Transforming an $n$-sided patch into a collection of 3 - and 4-sided patches 
Goldman [Gol83] has described techniques for computing the new rational Bézier representation for the four subtriangles. For corner subtriangles with a zero weight at a corner control point, a version of transformation 6 can convert triangular form to tensor product form. The result of these transformations is a rational Bézier representation without base points. With this representation, standard techniques for rendering or intersection testing can be applied to rational Bézier surfaces. The same construction can be applied to the weights at the vertices of a tensor product patch to create 5-, 6-, 7-, or 8-sided surface patches.

\subsection{Continuity Conditions}

A primary application of five- or six-sided Bézier surfaces is in the smooth filling of the holes that arise when blending together collections of rectangular patches. Figure 5 illustrates two common situations that arise when filleting the edges of several adjacent cubes. In such situations, orders of continuity at the intersection of triangular, rectangular, and multiplesided patches are important. This section considers the continuity conditions for smoothly meshing patches.

Simple $C^{0}$ continuity is straightforward. If control points with zero weight have been

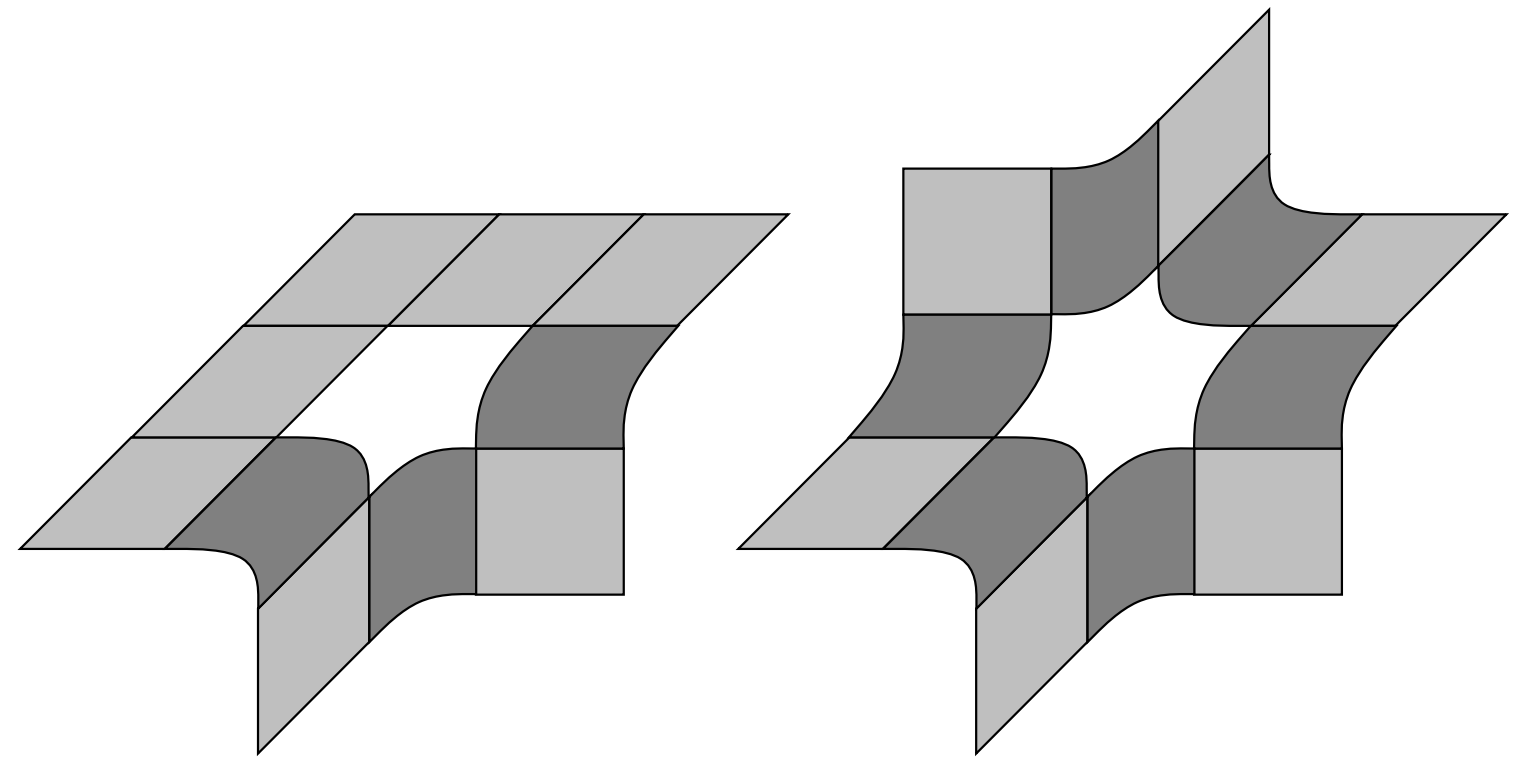

FIGURE 5: Five- and six-sided holes arising during filleting 
deleted, then the boundary curves of the resulting surface patch are rational Bézier curves whose control polygons correspond to boundary of the control polyhedron.

Conditions for $C^{1}$ continuity between two rational Bézier surface patches $S_{0}$ and $S_{1}$ in either triangular or tensor product form have been presented [Pip87, DeR90]. These conditions involve only those control points on the common boundary between $S_{0}$ and $S_{1}$, and those control points adjacent to the boundary. They can be summarized roughly as follows.

Let $E(t)$ be the rational Bézier curve shared by the two patches. Let $C(t)$ and $D(t)$ denote the rational Bézier curves defined by those control points adjacent to $E(t)$, but not defining it, on $S_{0}$ and $S_{1}$ respectively. Let $A(t)$ and $B(t)$ denote rational Bézier curves that result from deleting the first and last control points of $E(t)$. Figure 6 shows portions of the control polyhedra for $S_{0}$ and $S_{1}$, where the dashed control polygon identifies $E(t)$, and bold control polygons identify $A(t), B(t), C(t)$ and $D(t)$. Now, $S_{0}$ and $S_{1}$ share a common tangent plane along $E(t)$ if and only if $A(t), B(t), C(t)$, and $D(t)$ are coplanar for all $t$.

For the three edges of a multi-sided patch that are images of the sides of the domain triangle, these conditions can be applied directly if those control points with zero weight are removed and the weights of the remaining control points modified via equation 4 . For a boundary curve corresponding to a vertex of the domain triangle, the conditions can be

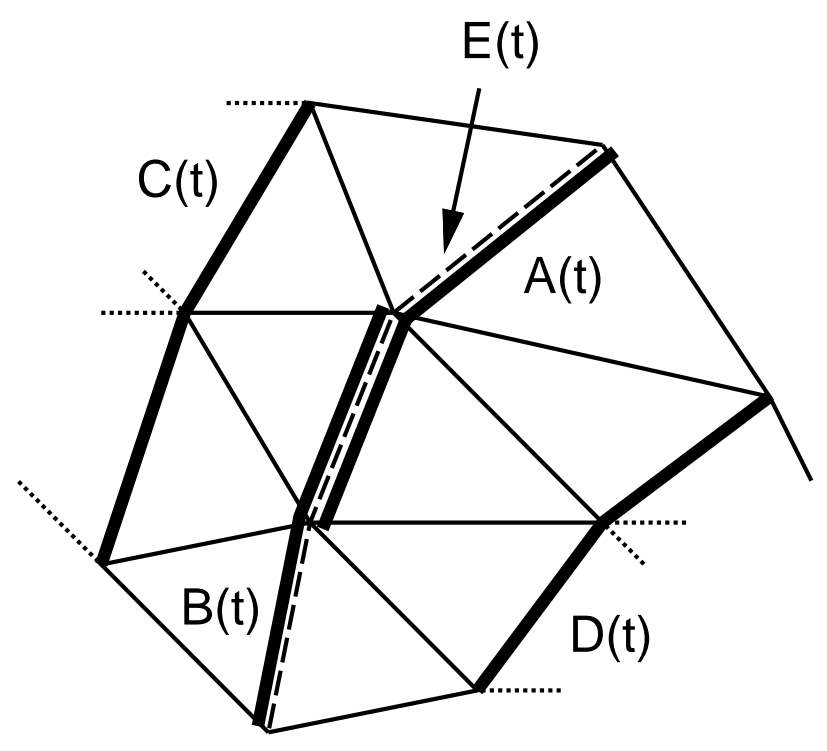

Figure 6: $C^{1}$ continuity conditions for rational patches 
directly applied to the rational Bézier curves defined by the closest two layers of control points. This generalization works because the new boundary curve of the patch is the boundary of the tensor product surface that results from transformation 6 .

We illustrate these conditions with several examples. Photo \#1 shows a rational Bézier surface of parametric degree six. It is formed by degree-raising the linear parameterization of a triangle to degree six, then perturbing one control point. The control point $\mathbf{p}_{222}$ does not affect $C^{1}$ continuity conditions for the boundary of the patch and has been moved in a direction normal to the plane containing the original triangle. The resulting patch remains tangent to the original plane along its boundary. Photo \#2 illustrates the six-sided patch that results when the surface patch of Photo \#1 has the following weights set to zero.

$$
\begin{aligned}
& w_{006}=w_{105}=w_{015}=0, \\
& w_{060}=w_{150}=w_{051}=0, \\
& w_{600}=w_{510}=w_{501}=0 .
\end{aligned}
$$

Again $\mathbf{p}_{222}$ does not affect $C^{1}$ continuity because it is adjacent to no control points along the boundary of the patch. Thus, the resulting patch must also be tangent to the plane containing the boundary curves. Photo \#3 shows a five-sided patch filling a hole that might

arise while filleting the edges of several adjacent blocks as in figure 5. Photo \#4 shows a six-sided patch filling a similar six-sided hole. Both patches have parametric degree six and meet their neighboring patches with tangent plane continuity.

\subsection{Understanding Gregory's Patch}

With our understanding of the effect of base points on rational surface patches, it is possible to describe Gregory's patch [Gre74] solely in terms of control points and weights. As mentioned in the introduction, Gregory defined a type of surface patch for $C^{1}$ boundary interpolation using a special class of rational basis functions. At a corner of the parametric domain, these basis functions evaluate to $\frac{0}{0}$. Thus, the resulting parameterization has a base point at this corner. Using limits, it is possible show that these functions and their first derivatives are always well-defined at the vertex. However, the value of the mixed partial derivatives at the vertex might depend on the order of computation of the various limits needed to evaluate the rational functions at that vertex. The effect on the surface patch is that the edge twists at the vertex of the patch might not agree along each incident edge. 
It is possible to describe Gregory's construction solely in terms of control points and weights. The rational functions that Gregory uses introduce a base point at the corner of the parametric domain. Let $\mathbf{p}_{00 n}$ and $w_{00 n}$ denote the control point and weight associated with this vertex. The existence of a base point at $(0,0,1)$ implies that $w_{00 n}=0$. As described above, the result of setting $w_{00 n}=0$ is the introduction of a new edge linking the control points $\mathbf{p}_{01(n-1)}$ and $\mathbf{p}_{10(n-1)}$. In Gregory's construction, the edge is collapsed back to a vertex by setting $\mathbf{p}_{01(n-1)}=\mathbf{p}_{10(n-1)}$. The tangent conditions are controlled by the next layer of control points, $\mathbf{p}_{02(n-2)}, \mathbf{p}_{11(n-2)}$, and $\mathbf{p}_{20(n-2)}$, so to guarantee that the resulting surface patch has a distinct tangent plane at $\mathbf{p}_{01(n-1)}$, Gregory forces these three control points to be collinear.

The product of this construction is a patch in which the value of the mixed partial derivatives in $\alpha$ and $\beta$ of the rational parametric functions at $(0,0,1)$ are not uniquely defined. In evaluating at $(0,0,1)$, one can first substitute $\alpha=0$, clear any common factors

in $\beta$, and then substitute $\beta=0$. The resulting value is the edge twist along the boundary curve $\alpha=0$. In terms of control points, this value is directly determined by $\mathbf{p}_{12(n-3)}$. Alternatively, one can substitute $\beta=0$, clear any common factors in $\alpha$, and substitute $\alpha=0$. This value is the edge twist along the boundary curve $\beta=0$. The control point $\mathbf{p}_{21(n-3)}$ controls this edge twist. Since $\mathbf{p}_{12(n-3)}$ and $\mathbf{p}_{21(n-3)}$ are distinct, this vertex of the Gregory patch can have distinct edge twists along each edge incident to the vertex. Figure 7 illustrates the construction.

\section{Future Work}

The preceding sections have described the effect of setting a triangle of weights to zero at one corner of the domain triangle of a rational Bézier surface. This technique allows creation of four-, five- or six-sided Bézier surfaces. In theory, it is possible to generalize this construction to create rational Bézier surfaces with arbitrarily many sides. The key to this construction is the Newton polygon of the parametric functions.

Let $F$ be a bivariate polynomial in Bernstein basis.

$$
F(\alpha, \beta, \gamma)=\sum_{\substack{i, j, k>0 \\ i+j+k=n}} a_{i j k} B_{i j k}(\alpha, \beta, \gamma)
$$

The Newton polygon of $F$ is the convex hull of $\left\{(i / n, j / n, k / n) \mid a_{i j k} \neq 0\right\}$ [Wa150]. If $S$ is 


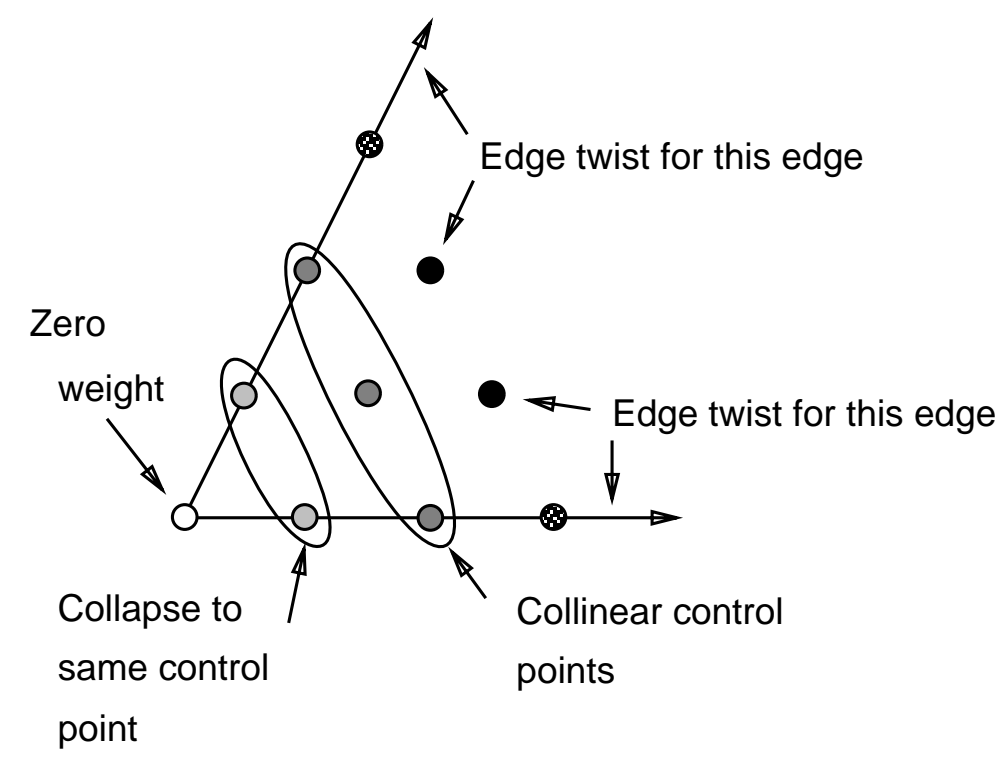

FIGURE 7: The structure of a Gregory patch near a vertex

a rational Bézier surface as defined in equation 2, then the Newton polygon for the homogeneous parameter $w$ provides the key to understanding the structure of $S$. Note that the Newton polygons for $x, y$, and $z$ are contained in the Newton polygon for $w$ because the coefficient $\mathbf{p}_{i j k}$ is scaled by $w_{i j k}$. The Newton polygon of $w$ is called the Newton polygon associated with $S$.

Many of the geometric properties of a rational surface are captured by its associated Newton polygon. For example, the edges of a rational Bézier surface are in one-to-one correspondence with the edges of its associated Newton polygon [War91b]. Unfortunately, the proof techniques used in [War91b] give no insight into the behavior of the rational surface patch away from its boundary. Further work incorporating techniques from toric variety theory [Oda85, KKMSD73] may lead to practical methods for rendering, subdividing and meshing patches with seven or more sides.

Another interesting property of Newton polygons is that the implicit degree of a rational surface is bounded by the area of its associated Newton polygon [War91a]. In future work, we hope to use this observation in creating rational surfaces with low implicit degree. 


\section{Acknowledgments}

I would like to thank Ron Goldman and Eng-Wee Chionh for their initial discussion on the topic of base points on rational surfaces. I would also like to thank Jörg Peters for

bringing to my attention the existence of base points on Gregory's patch. Finally, I would like to thank the anonymous referees for their helpful comments.

\section{References}

[Chi90] Eng-Wee Chionh. Base Points, Resultants, and the Implicit Representation of Rational Surfaces. PhD thesis, University of Waterloo, 1990.

[DeR90] Tony DeRose. Necessary and sufficient conditions for tangent plane continuity of Bézier surfaces. Computer Aided Geometric Design, 7(1-4):165-180, June 1990.

[Far88] Gerald Farin. Curves and Surfaces for Computer Aided Geometric Design: A Practical Guide. Academic Press Inc., New York, 1988.

[Go183] Ron Goldman. Subdivision algorithms for Bézier triangles. Computer-aided Design, 15:159-166, January 1983.

[Gre74] J. A. Gregory. Smooth interpolation without twist constraints. In R. E. Barnhill and R. F .Riesenfeld, editors, Computer Aided Geometric Design, pages 71-88. Academic Press, New York, 1974.

[Gre83a] J. A. Gregory. $C^{1}$ rectangular and non-rectangular surface patches. In R. E. Barnhill and W. Boehm, editors, Surfaces in CAGD, pages 25-33. NorthHolland, 1983.

[Gre83b] J. A. Gregory. n-sided surface patches. In J. A. Gregory, editor, Mathematics of Surfaces, pages 217-232. Clarendon Press, Oxford, 1983.

[HK84] M. Hosaka and F. Kimura. Non-four-sided patch expressions with control points. Computer Aided Geometric Design, 1(1):75-86, 1984. 
[KKMSD73] G. Kempf, F. Knudsen, D. Mumford, and B. Saint-Donat. Toroidal Embeddings I. Lecture Notes in Mathematics 339. Springer Verlag, 1973.

[LD89] Charles Loop and Tony DeRose. A multisided generalization of Bézier surfaces. ACM Transactions on Graphics, 8(3), 1989.

[Oda85] T. Oda. Convex Bodies and Algebraic Geometry. Springer-Verlag, 1985.

[Pip87] B. Piper. Visually smooth interpolation with triangular Bézier patches. In Gerald Farin, editor, Geometric Modeling: Algorithms and New Trends, pages 221-234. SIAM, 1987.

[Sab83] M. Sabin. Non-rectangular surface patches suitable for inclusion in a B-spline surface. In P. ten Hagen, editor, Proceedings of Eurographics 1983, pages 57-69, Amsterdam, 1983. North-Holland.

[Wa150] R. Walker. Algebraic Curves. Princeton University, 1950.

[War91a] Joe Warren. A bound on the degree of polygonal Bézier surfaces. In C. Bajaj, editor, Algebraic Geometry and Applications. Springer Verlag, 1991.

[War91b] Joe Warren. Multi-sided rational Bézier surfaces and newton polygons. In preparation, 1991. 